- the blood-thinner heparin, warns Paulo Mourão, a molecular biologist at the Federal University of Rio de Janeiro in Brazil.

The drug's active ingredient is extracted from the mucous membrane of pig intestines, and used to treat heart attacks, among other things; China accounts for almost $80 \%$ of worldwide production ${ }^{2}$. Swine fever "has the potential to cause an unprecedented shortage of heparin's raw material, which may jeopardize the global supply", says Mourão.

The Chinese government says the outbreaks are under control, but some researchers are sceptical. There is little information about how the disease is spreading in China, or how effective the control measures have been, says Linda Dixon, a virologist at the Pirbright Institute, UK. She suspects that some cases might have gone undetected.

China has roughly 440 million pigs, and unofficial estimates of the proportion currently infected with the virus vary from $10 \%$ to $40 \%$, says Dirk Pfeiffer, an epidemiologist at the City University of Hong Kong.

The government is prioritizing research on the virus, setting aside about 100 million yuan (US\$15 million) for projects, says George Gao, vice-president of the National Natural Science Foundation of China in Beijing.

The work will help to fill the many gaps in scientists' understanding of the virus, including its detailed structure and how it transmits to hosts and evades their immune systems. "We know almost nothing about this virus," says Gao.
Before the outbreaks, researchers in China were banned from studying the live virus in case it escaped from a lab and infected pigs. Now that the infection has entered the country, scientists at the second-highest-level biosafety laboratories can study it in cells, and those at the most secure biosafety laboratory can work with it in animals, says Gao. "Within a couple of months, I expect to see Chinese scientists contributing a lot to the field."

\section{VACCINE DEVELOPMENT}

The Harbin Veterinary Research Institute (HVRI), part of the Chinese Academy of Agricultural Sciences, is among the few institutions in the country with permission to handle the live virus in cell culture, and the only facility qualified to test the infection in pigs, which is essential for vaccine development, says $\mathrm{Bu}$ Zhigao, who heads the institute.

Researchers at the HVRI are investigating what, if any, natural immunity pigs have to swine fever, with a view to making drugs and developing new diagnostic techniques. But the priorities are learning how quickly the virus spreads and developing vaccines, says Zhigao, although he declined to comment on the details of the research.

At a symposium on swine fever in Beijing on 9 April, Pfeiffer says, HVRI scientists presented research on a potential vaccine that triggers an immune response using a less harmful strain of the pathogen - known as an attenuated vaccine - instead of an inactivated strain. Attenuated vaccines tend to produce a stronger and longer-lasting immune response than inactive vaccines, says Dixon.

But Pfeiffer and Dixon are concerned about the intense political pressure in the country to come up with a vaccine quickly.

With live-attenuated vaccines, there is a risk that the microorganism could persist and spread through pigs, and cause unacceptable adverse reactions, Pfeiffer says. "It is a bit like playing with fire."

One thing to monitor is whether a vaccine prevents replication of the deadly form of the virus in a host, notes Dixon. If it doesn't, vaccinated pigs, which might show no symptoms, could infect unvaccinated animals. A vaccine is probably still some years off, says Dixon.

Groups in China are also studying how the virus survives in the host. In February, researchers at the HVRI reported that a strain extracted from the spleen of an infected pig was extremely virulent, resulting in certain death, and efficient at passing from pig to $\mathrm{pig}^{3}$.

These results will help to improve measures to prevent and control the disease, says Zhigao, the HVRI paper's lead author. "We must control this disease, no matter how big a cost it is," he says.

\footnotetext{
1. Bao, J. et al. Transbound. Emerg. Dis. https://doi. org/10.1111/tbed.13124 (2019).

2. Vilanova, E., Tovar, A. M. F. \& Mourão, P. A. S. J. Thromb. Haemost. 17, 254-256 (2018).

3. Zhao, D. et al. Emerg. Microbes Infect. 8, 438-447 (2019).
}

\section{BY AMY MAXMEN}

$\mathrm{W}$ hen health workers in Malawi began rolling out the first vaccine proven to protect against malaria, it was a moment 32 years - and more than US\$700 million — in the making.

The country began giving the vaccine, called RTS,S, to children under the age of two on 23 April. Ghana and Kenya will soon join in, as part of a large-scale pilot programme backed by the World Health Organization (WHO). The effort could immunize more than one million children by 2023 - a welcome boost in the fight against malaria, which kills an average of 1,200 people worldwide per day. The vast majority are children in Africa.
"We are excited and support this vaccine since there is nothing else," says Michael Kayange, a programme manager at Malawi's National Malaria Control Programme in Lilongwe.

But the plan is not without controversy. The RTS,S vaccine prevents only 4 in 10 malaria cases, and must be given as 4 injections over 18 months. Some malaria researchers question the wisdom of spending time and money on the pilot programme, given that a handful of more effective vaccines are in clinical trials and could be available by the time RTS,S is ready for regular use. They say that the decades-long effort to roll out RTS,S exemplifies the plodding progress on vaccines for diseases that affect the world's poor, and highlights the need for a more efficient path forward.

"We need to rethink this whole process," says James Tibenderana, global technical director at the Malaria Consortium in London. "We can't expect pharma to spend another 30 years making a vaccine that will then be piloted for 3-4 years before people make their mind up about that result."

Officials at GlaxoSmithKline (GSK), the London-based pharmaceutical firm that manufactures the vaccine, agree. "We are incredibly proud to see it rolled out," says Thomas Breuer, the company's chief medical officer for vaccines. But, he adds, "This kind of endeavour can't be repeated, from GSK's point of view - we have to find other ways."

When scientists at the Walter Reed 
the WHO in Geneva, Switzerland, says that researchers will compare the health of vaccinated children in Malawi, Ghana and Kenya with that of unvaccinated kids in neighbouring regions. "We will be watching for imbalances in deaths, severe malaria and meningitis," she says. The WHO's models suggest that one life will be saved for every 200 children who are immunized.

That means the pilot could save thousands of people. But Sabra Klein, a public-health researcher at Johns Hopkins University in Baltimore, Maryland, worries that the cause of any safety issues that arise will be hard to identify, because the pilot is not a controlled trial. "We need to address this to improve the science behind our vaccines," she says.

Others, such as Adrian Hill, a vaccine researcher at the Jenner Institute in Oxford, UK, argue that the money for the pilot programme could be better spent on supporting the development of more effective vaccines; Hill is helping to develop a malaria vaccine called R21 that is set to begin phase II trials in Burkina Faso later this year. And some scientists want to re-engineer RTS,S to better defend against the most common strains of malaria parasite in Africa ${ }^{4}$.

"Someone needs to take a hard look at how money is being spent," Hill says. "There isn't a very independent entity that can ask if, in the current environment, it's worth putting another $\$ 52$ million into a vaccine designed in 1987." culminated in 2015, when a 15,000-person clinical trial found ${ }^{1}$ that giving 4 doses of RTS,S over 18 months cut the number of malaria episodes that young children experienced by $36 \%$.

But data from an earlier trial suggest that that partial protection fades over several years ${ }^{2}$. And a deep dive into data from the 2015 trial

revealed that girls who had received the RTS,S vaccine were slightly more likely than those ding severe malaria ${ }^{3}$. At the sam Tibenderana says, officials at malaria-control agencies in Africa worried about how to afford gle to deploy bed nets and drugs to treat the infection.

Mindful of such concerns, the WHO recommended in 2015 that the RTS,S vaccine be rolled out in phases, to identify any safety issues and to determine the feasibility of wider distribution. In 2016, three non-profit health groups - the Global Fund to Fight AIDS, Tuberculosis and Malaria, the vaccine alliance Gavi, and Unitaid - agreed to fund a pilot programme for four years, at a cost of $\$ 52$ million.

Mary Hamel, a medical epidemiologist at
1. RTS,S Clinical Trials Partnership. Lancet 386 31-45 (2015).

2. Olotu, A. et al. N. Engl. J. Med. 374, 2519-2529 (2016).

3. Klein, S. L., Shann, F., Moss, W. J., Benn, C. S. \& Assby, P. mBIO 7, e00514-16 (2016).

4. Neafsey, D. E. et al. N. Engl. J. Med. 373, 2025-2037 (2015).

\section{Black hole possibly ate neutron star}

\section{Gravitational-wave detectors spot what might be the first evidence of such an event, as their activity picks up.}

\section{BY DAVIDE CASTELVECCHI}

$\mathrm{T}$ The newly upgraded detectors of the LIGO and Virgo gravitational-wave observatories have sent astronomers on a wild ride. Last week, the detectors heard gravitational waves from a merger of two neutron stars for only the second time - creating a frenzy of activity. Just a day later, the observatories made the first possible sighting of a black hole devouring a neutron star. If confirmed, it would be the first evidence that the Universe harbours such binary systems. Observations of their dramatic collisions can produce a wealth of information that no other event could provide, including precise tests of the general theory of relativity and measuring the Universe's rate of expansion.

On 25 April, a train of waves hit one of the two detectors of the Laser Interferometer Gravitational-wave Observatory (LIGO), in Livingston, Louisiana, and the Virgo observatory, near Pisa, Italy. (At the time, LIGO's second machine, in Hanford, Washington, was briefly out of commission.) That event was a clear-cut case of two merging neutron stars, and came nearly two years after the historic discovery of such an event in August 2017. The researchers estimated that the collision occurred some 150 megaparsecs (500 million light years) away, around three times farther than the 2017 merger.

A day later, on 26 April, another train of waves hit both LIGO detectors and Virgo. So far, researchers have performed only a preliminary analysis of that event, but they are considering the tantalizing prospect that they have detected a merger of a black hole and a neutron star.

"I think that the classification is leaning towards neutron star-black hole" merger, says Chad Hanna, a member of LIGO's data-analysis team and a physicist at Pennsylvania State University in University Park. 